\title{
Epigenetic regulation of telomere length in mammalian cells by the Suv39h1 and Suv39h2 histone methyltransferases
}

\author{
Marta García-Cao ${ }^{1}$, Roderick O’Sullivan ${ }^{2}$, Antoine H F M Peters ${ }^{2}$, Thomas Jenuwein $^{2}$ \& María A Blasco ${ }^{1}$
}

Telomeres are capping structures at the ends of eukaryotic chromosomes composed of TTAGGG repeats bound to an array of specialized proteins ${ }^{1-3}$. Telomeres are heterochromatic regions. Yeast and flies with defects in activities that modify the state of chromatin also have abnormal telomere function ${ }^{4-6}$, but the putative role of chromatin-modifying activities in regulating telomeres in mammals is unknown. Here we report on telomere length and function in mice null with respect to both the histone methyltransferases (HMTases) Suv39h1 and Suv39h2 (called SUV39DN mice). Suv39h1 and Suv39h2 govern methylation of histone $\mathrm{H} 3$ Lys9 (H3-Lys9) in heterochromatic regions ${ }^{7}$. We show that primary cells derived from SUV39DN mice have abnormally long telomeres relative to wild-type controls. Using chromatin immunoprecipitation (ChIP) analysis, we found that telomeres were enriched in diand trimethylated H3-Lys9 but that telomeres of SUV39DN cells had less dimethylated and trimethylated $\mathrm{H} 3$-Lys9 but more monomethylated H3-Lys9. Concomitant with the decrease in $\mathrm{H} 3$-Lys9 methylation, telomeres in SUV39DN cells had reduced binding of the chromobox proteins $\mathrm{Cbx} 1, \mathrm{Cbx} 3$ and $\mathrm{Cbx} 5$, homologs of Drosophila melanogaster heterochromatin protein 1 (HP1). These findings indicate substantial changes in the state of telomeric heterochromatin in SUV39DN cells, which are associated with abnormal telomere elongation. Taken together, the results indicate epigenetic regulation of telomere length in mammals by Suv39h1 and Suv39h2.

The N-terminal tails of histones are subjected to post-translational modifications, including acetylation, methylation and phosphorylation, generating an extensive repertoire of chromatin structures ${ }^{8,9}$. Binding of factors that specifically recognize these modified histones mediate cellular responses ${ }^{8,9}$. Heterochromatic regions are enriched in methylated H3-Lys9, which creates a binding site for the chromobox proteins ${ }^{9}$, mediators of heterochromatin formation and epigenetic gene regulation. In D. melanogaster, HP1 is also required for telomere capping ${ }^{4,5}$. To investigate the impact of H3-Lys9 methylation on telomere length regulation and telomere function, we studied embryonic stem (ES) cells and mouse embryonic fibroblasts (MEFs) from SUV39DN mice ${ }^{7}$, which have less methylation of H3-Lys9 at the pericentric heterochromatin compared to wildtype mice ${ }^{7}$. First, we used terminal restriction fragment (TRF) analysis to estimate telomere length in these cells. Two independent SUV39DN ES cell cultures showed abnormally long telomeres, which were not present in wild-type ES cells (Fig. 1a). This finding was confirmed using MEFs (passage 1-2) derived from SUV39DN and wild-type littermate embryos (Fig. 1a). TRF analysis of increasing passages of the SUV39DN MEFs (D43) showed that the long telomeres in these MEFs were stable (Fig. 1b).

Next, we carried out quantitative fluorescence in situ hybridization (Q-FISH) using a telomere-specific peptide nucleic acid probe. We determined the average telomere length for each ES-cell and MEF culture (Table 1) and the corresponding telomere length distributions (Fig. 1c,d). SUV39DN ES cells and MEFs showed a significant proportion of very long telomeres at both $\mathrm{p}$ and $\mathrm{q}$ chromosome arms, which were not present in the wild-type controls (Fig. 1c,d). Student's $t$-test analyses comparing all individual telomere length values indicated that the differences were significant $(P<0.001)$.

Analysis of each individual metaphase confirmed that some telomeres were abnormally long in the SUV39DN ES cells and MEFs compared with the controls (Fig. 2a,b). To identify the chromosomes with abnormally long telomeres in SUV39DN cells, we used spectral karyotyping analysis (SKY) to identify chromosomes combined with telomeric Q-FISH to determine telomere length. Combined Q-FISH and SKY indicated that the same set of chromosomes generally had abnormally long telomeres in a given MEF culture (Table 2 and Fig. 2b,c). Thus, deletion of the HMTases Suv39h1 and Suv39h2 results in deregulation of telomere length in random sets of chromosomes, which are maintained in culture and whose identity varies depending on the MEF culture. In the case of ES cells, however, the chromosomes with abnormally long telomeres varied between different metaphases of the same culture (Table 2 and Fig. 2a,d), suggesting differences between ES cells and MEFs in response to abrogation of Suv39h1 and Suv39h2. Q-FISH analysis also

${ }^{1}$ Molecular Oncology Program, Spanish National Cancer Centre (CNIO), E-28029 Madrid, Spain. ${ }^{2}$ Research Institute of Molecular Pathology, Vienna Biocenter, A1030 Vienna, Austria. Correspondence should be addressed to M.A.B. (mblasco@cnio.es). 
showed that the long telomeres present in SUV39DN cells were preserved at both sister chromatids in a given chromosome arm (Fig. $\mathbf{2 c}, \mathbf{d}$ ), indicating that they were generated before or during DNA replication and that there were no differences between the telomeres of the leading and lagging strands.

a

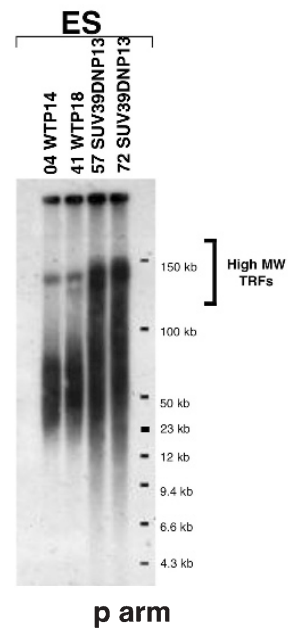

C

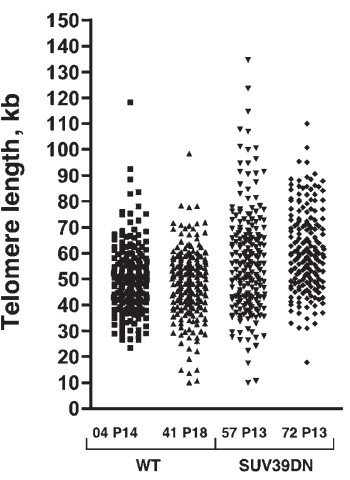

p arm

d

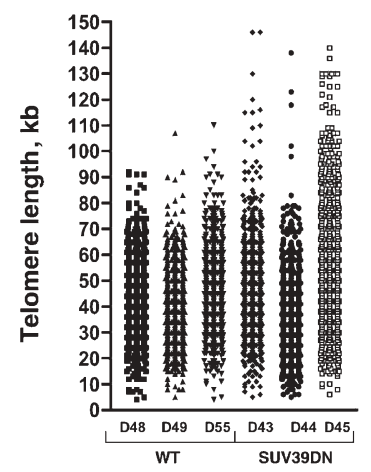

b
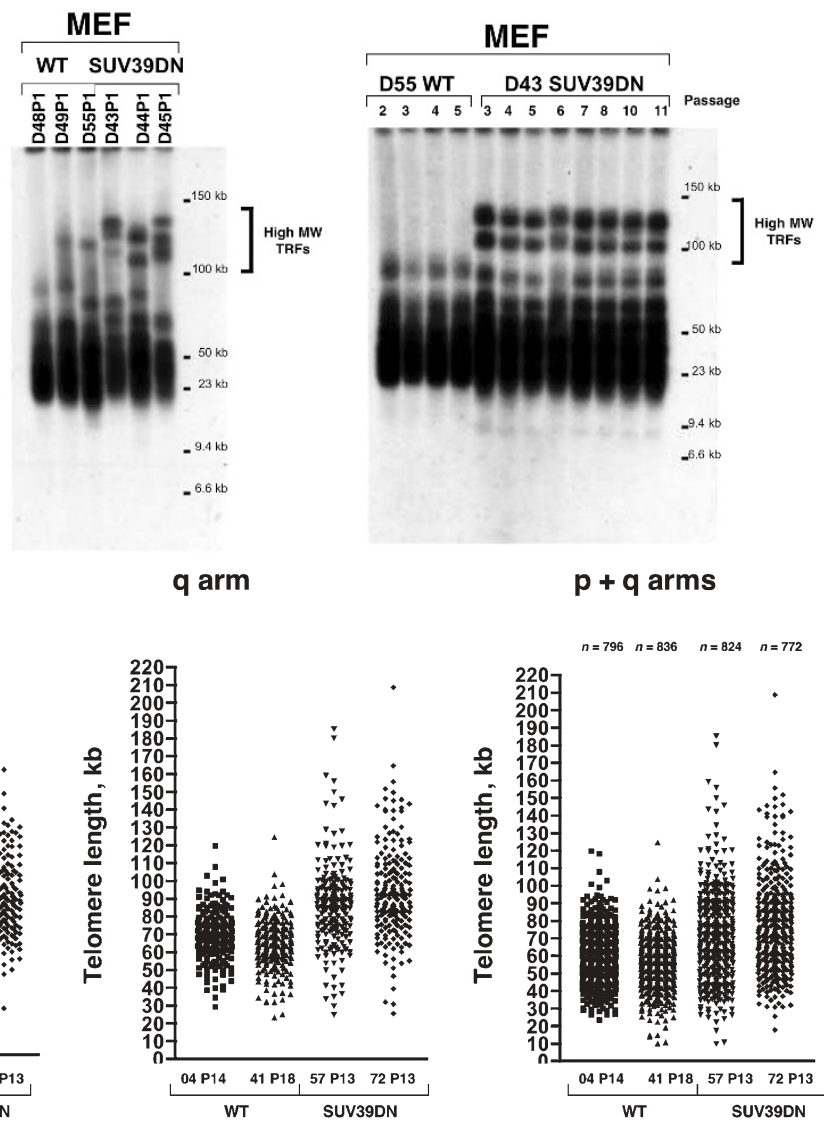

q arm

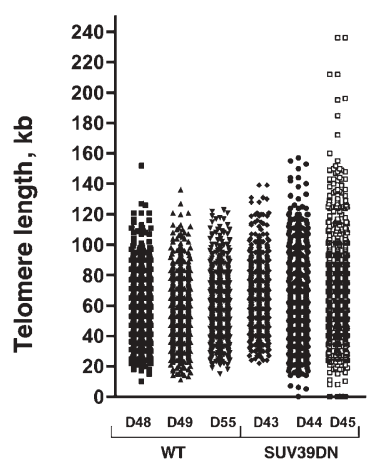

We next examined whether the abnormally long telomeres present in the SUV39DN cells retained their end-capping function. Dysfunctional telomeres have been shown to result in more end-toend chromosome fusions ${ }^{10-15}$. Robertsonian-like (RT-like) fusions are the most common structural aberration associated with telomere dysfunction in mouse cells ${ }^{10-12,14,15}$. No RT-like fusions, however, were detected in the SUV39DN cells after scoring $\geq 40$ metaphases by Q-FISH from each ES-cell and MEF culture, in agreement with previous results ${ }^{7}$, suggesting that telomeres retained their protective function in these cells (Supplementary Tables $\mathbf{1}$ and $\mathbf{2}$ online). In addition, none of the SUV39DN ES-cell or MEF cultures had higher frequencies of undetectable telomeres (Table 1), a hallmark of telomere dysfunction ${ }^{14,15}$. The SUV39DN MEFs, however, had higher frequencies of chromatid breaks, chromatid fusions and metaphases with complex chromosomal rearrangements (radial chromosomes, translocations) relative to wild-type MEFs (Supplementary Table 1 and Supplementary Fig. 1 online). Notably, the chromosomes harboring abnormally long telomeres were not involved in the chromosomal aberrations present in SUV39DN cells as determined by combined Q-FISH and SKY analysis (Supplementary Table 2 online). The SUV39DN cells also had more polyploid metaphases than did the controls (Supplementary Fig. 2 online), including the presence of butterfly chromosomes (Supplementary Fig. 1 online). Taken together, these findings agree with the role of Suv39h1 and Suv39h2 in genomic stability $^{7}$ and indicate that the abnormally long telomeres in SUV39DN cells retain a normal capping function.

To address whether the effect on telomere length was due to changes in expression of telomerase, we carried out telomeric repeat amplification protocol assays in wild-type and SUV39DN cells. Independent SUV39DN EScell and MEF cultures and wild-type controls had similar levels of telomerase activity (data not shown), suggesting that the telomere elongation phenotype is not associated with changes in telomerase activity. It is possible, however, that the accessibility of telomerase to the telomere is altered in SUV39DN cells due to changes in the telomeric chromatin.

In this regard, our results suggest that Suv39h1 and Suv39h2, known to be involved in maintaining pericentric heterochromatin ${ }^{7}$, may also have a role in maintaining telomeric heterochromatin. To address this, we studied whether mammalian telomeres contained features of constitutive heterochromatin, such as methylation of H3-Lys9 and recruitment of chromobox proteins Cbx1, Cbx3 and Cbx5.

Figure 1 Telomere length determination in SUV39DN MEF and ES cells. (a) Representative TRF analysis of two wild-type (WT) and two SUV39DN ES-cell cultures and of three wild-type and three SUV39DN MEF cultures. Passage number is indicated (P). (b) TRF analysis of wild-type (WT) and SUV39DN MEFs at different passages. (c) Telomere length distribution of $p$ telomeres, $q$ telomeres and the sum of $p+q$ telomeres in independent wild-type (WT) and SUV39DN ES-cell cultures as determined by Q-FISH. (d)Telomere length distribution of $p$ telomeres, $q$ telomeres and the sum of $p+$ q telomeres in independent wild-type (WT) and SUV39DN MEF cultures as determined by Q-FISH. 
Table 1 Telomere length in wild-type and SUV39DN cells as determined by Q-FISH

\begin{tabular}{|c|c|c|c|c|c|c|c|}
\hline Cell type & $\begin{array}{l}\text { Genotype, culture } \\
\text { and passage }(P)\end{array}$ & $\mathrm{p}$ arm (kb) & $\mathrm{q}$ arm (kb) & $\begin{array}{l}\text { Average } p+q \\
(k b)\end{array}$ & $\begin{array}{l}\text { Metaphases } \\
\text { analyzed }\end{array}$ & $\begin{array}{l}\text { Number of } \\
\text { telomeres }\end{array}$ & $\begin{array}{c}\text { Percentage of } \\
\text { undetectable } \\
\text { telomeres } \\
\text { (signal-free ends) }\end{array}$ \\
\hline ES & Wild-type 04P14 & $50.0 \pm 13.2$ & $69.8 \pm 14.0$ & $59.6 \pm 13.6$ & 5 & 796 & 0 \\
\hline ES & Wild-type 41P18 & $48.4 \pm 13.1$ & $64.7 \pm 14.5$ & $56.5 \pm 13.8$ & 5 & 836 & 0 \\
\hline ES & SUV39DN 57P13 & $56.7 \pm 19.8$ & $86.5 \pm 23.7$ & $71.6 \pm 21.8$ & 5 & 824 & 0 \\
\hline ES & SUV39DN 72P13 & $60.9 \pm 15.5$ & $93.7 \pm 25.9$ & $77.2 \pm 20.7$ & 5 & 772 & 0 \\
\hline MEF & Wild-type D48P1 & $41.1 \pm 14.6$ & $59.9 \pm 20.9$ & $50.5 \pm 17.8$ & 10 & 1544 & 0 \\
\hline MEF & Wild-type D49P1 & $37.8 \pm 14.1$ & $54.0 \pm 20.6$ & $45.9 \pm 17.3$ & 10 & 1564 & 0 \\
\hline MEF & Wild-type D55P1 & $45.7 \pm 15.2$ & $57.6 \pm 19.3$ & $51.7 \pm 17.2$ & 10 & 1576 & 0 \\
\hline MEF & SUV39DN D43P2 & $46.7 \pm 18.9$ & $64.9 \pm 20.1$ & $55.8 \pm 19.5$ & 10 & 1556 & 0 \\
\hline MEF & SUV39DN D44P1 & $36.3 \pm 16.0$ & $58.1 \pm 25.8$ & $47.2 \pm 20.9$ & 10 & 1568 & $0.06 \%$ \\
\hline MEF & SUV39DN D45P1 & $53.8 \pm 22.6$ & $69.7 \pm 28.9$ & $61.7 \pm 25.7$ & 10 & 2040 & $0.2 \%$ \\
\hline
\end{tabular}

Average telomere length was significantly higher in SUV39DN ES cells and MEFs than in the corresponding wild-type controls $(P<0.001$ by student's $t$-test comparing $\sim 1,500$ and $\sim 4,500$ telomere values from each genotype for ES cells and MEFs, respectively).

We carried out ChIP assays combined with dot-blot analysis to detect telomeric TTAGGG repeats. Telomeres of wild-type MEFs and ES cells had high levels of methylated H3-Lys9 (as detected with an antibody against 'branched' methylated H3-Lys9; ref. 7; Fig. 3a) and trimethylated H3-Lys9 (Fig. 3b) but no trimethylated H3-Lys27. Methylation of
H3-Lys9 at telomeres was markedly less in SUV39DN MEF and ES cells than in corresponding wild-type controls (Fig. 3a,b). Methylation of $\mathrm{H} 3$-Lys9 at centromeric repeats, which we measured as a control, was also less in SUV39DN cells than in corresponding wild-type cells (Fig. $3 \mathbf{a}$ and Supplementary Fig. 3 online), as previously described ${ }^{16}$. a

57 P13 SUV39DN

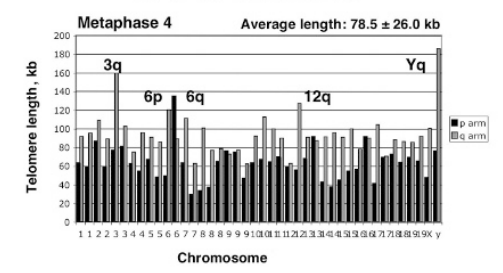

b D43 P2 SUV39DN

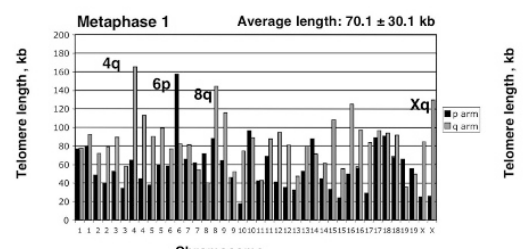

C

Metaphase 5

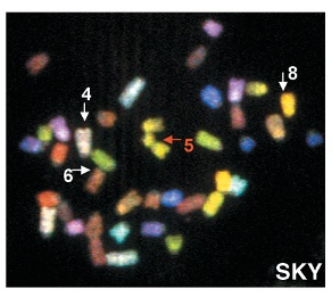

04 P14 WT

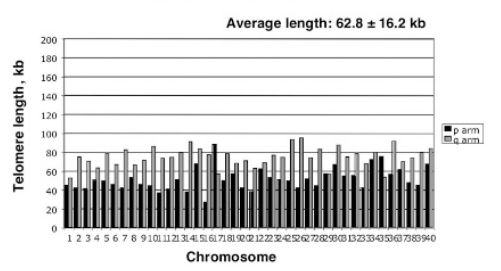

D49 P1 WT

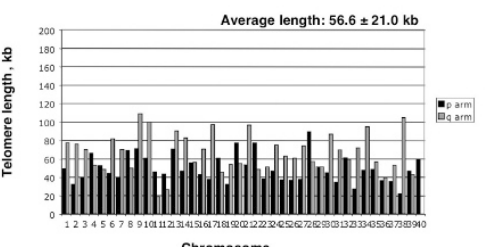

Figure 2 Identification of chromosomes harboring very long telomeres in SUV39DN cells. (a,b) Telomere length distributions of individual metaphases derived from the indicated SUV39DN ES-cell (a) and MEF (b) cultures and from the wild-type controls. Average telomere length of all telomeres from each metaphase is indicated in kb. $p$ and $q$ arms are shown in black and gray, respectively. Chromosomes with abnormally long telomeres (see also Table 2) are indicated in the histogram. (c,d) Representative metaphases of the indicated MEF (c) and ES-cell (d) cultures after combined SKY and Q-FISH. The histogram or 'skyline' of the metaphase shows telomere length for each individual chromosome in the metaphase. $p$ and $q$ arms are shown in black and gray, respectively. The asterisks in the skyline indicate the chromosomes that have abnormally long telomeres in the SUV39DN ES-cell and MEF cultures (see also Table 2). White arrows point to abnormally long telomeres in the Q-FISH and SKY images. The red arrow in (c) indicates a break at chromosome 5.

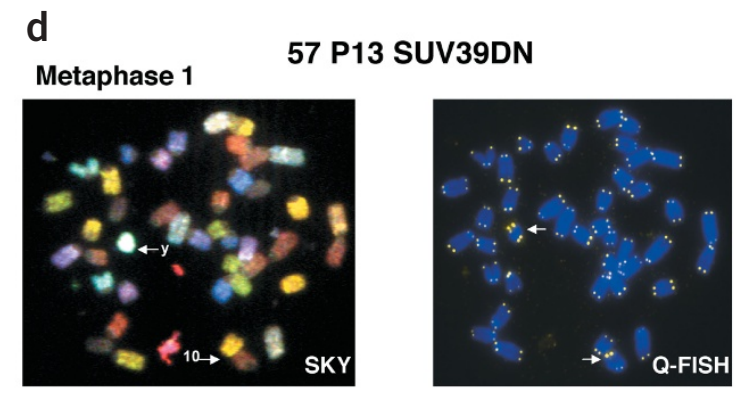

Skyline of the metaphase

Skyline of the metaphase
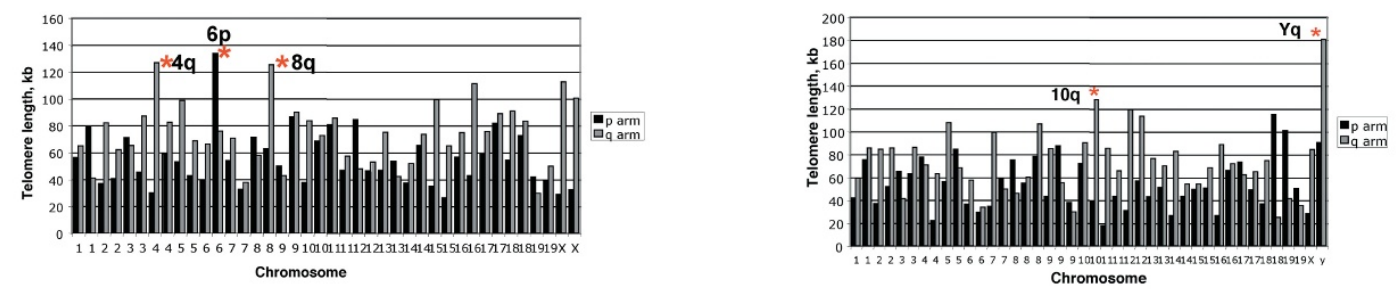
Table 2 Identification of chromosomes harboring very long telomeres in SUV39DN ES cells and MEFs using combined Q-FISH and SKY

\begin{tabular}{|c|c|c|c|c|c|c|}
\hline Cell type & Genotype & Metaphase 1 & Metaphase 2 & Metaphase 3 & Metaphase 4 & Metaphase 5 \\
\hline ES & SUV39DN 57p13 & $10 q, Y q$ & $8 q, 10 q, 12 q, 13 q$ & $6 p, 8 q, 12 q, Y q$ & $3 q, 6 p, 6 q, 12 q, Y q$ & $3 q, 5 q, Y q$ \\
\hline MEF & SUV39DN D43p2 & $4 q, 6 p, 8 q, X q$ & $4 q, 6 p, 8 q$ & $6 p, 8 q$ & $6 p, 8 q$ & $4 q, 6 p, 8 q$ \\
\hline MEF & SUV39DN D44p1 & $\begin{array}{c}12 q, 13 q \\
19 p, Y q\end{array}$ & $\begin{array}{c}12 q, 13 q \\
19 q, X q, Y q, N D\end{array}$ & $\begin{array}{l}12 q, 13 q \\
19 q, X q, Y q\end{array}$ & $\begin{array}{c}10 q, 12 q \\
19 p, Y q\end{array}$ & $\begin{array}{c}10 q, 2 \times 12 q, \\
2 \times 19 q, Y p, N D, N D\end{array}$ \\
\hline MEF & SUV39DN D45p1 & $3 q, 12 q, X q$ & $\begin{array}{c}3 q, 10 p \\
12 q, 2 \times X q\end{array}$ & $3 q, 12 q, X q$ & $\begin{array}{c}2 \times 3 q, 12 q \\
2 \times 13 q, 2 \times X q\end{array}$ & $\begin{array}{l}3 q, 12 q \\
13 q, X q\end{array}$ \\
\hline
\end{tabular}

ND, chromosome not determined by SKY; $p, p$ arm; q, q arm.

For ES cells, telomeres containing $\geq 120 \mathrm{~kb}$ of TTAGGG repeats are shown. For MEFs, telomeres containing $\geq 125 \mathrm{~kb}$ of TTAGGG repeats are shown.

Each lysine residue that can be methylated can be mono-, di- or trimethylated, extending the coding potential of histone $\mathrm{H} 3$ modification. Using antibodies highly specific to methylated lysine, we previously found that methylation of pericentric chromatin was not completely lost in SUV39DN cells but that the degree of methylation changed ${ }^{16}$. In wild-type cells, pericentric chromatin selectively accumulated trimethylated H3-Lys9, whereas in SUV39DN cells, it accumulated monomethylated H3-Lys9 and trimethylated H3-Lys27 (ref. 16). We show here that telomeres of wild-type cells were enriched in di- and trimethylated H3-Lys9, whereas telomeric chromatin in SUV39DN cells accumulated monomethylated H3-Lys9 and had less di- and trimethylated H3-Lys9 (Fig. 3d). Trimethylated H3-Lys27 was not more abundant at telomeric heterochromatin in SUV39DN cells compared to wild-type cells (Fig. $3 \mathbf{b}$ ), in contrast to its greater abundance at pericentric heterochromatin in these SUV39DN cells ${ }^{16}$ (Supplementary Fig. 3 online).

Methylation of histone $\mathrm{H} 3$ at Lys9 creates a binding site for chromobox proteins ${ }^{9}$, which mediate heterochromatin formation. ChIP analysis showed that wild-type telomeres contained $\mathrm{Cbx} 1, \mathrm{Cbx} 3$ and Cbx5, whereas Cbx5, Cbx3 and, to a lesser extent, Cbx1 were less abundant at SUV39DN telomeres (Fig. 3c), suggesting a loss of heterochromatic features. Taken together, these results indicate that the structure of mammalian telomeric chromatin shares many features with that of pericentric heterochromatin such as trimethylation of $\mathrm{H} 3$ Lys 9 and binding of chromobox proteins and that these modifications are maintained by the HMTases Suv39h1 and Suv39h2.

ChIP analysis also showed that wild-type telomeres contained TERF2 and TERF1 (Fig. 3a,e), telomere-binding proteins with roles a TTAGGG probe

Minor satellite probe

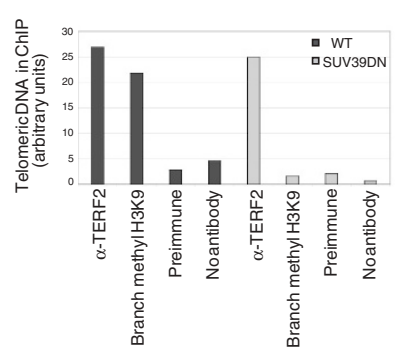

b

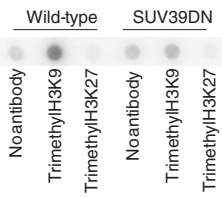

C

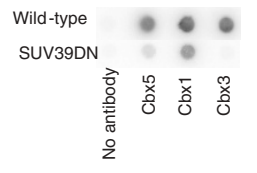

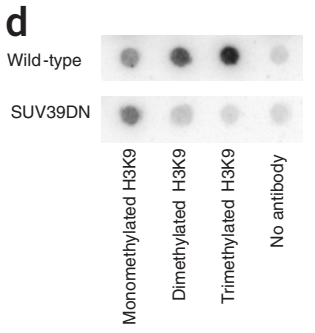

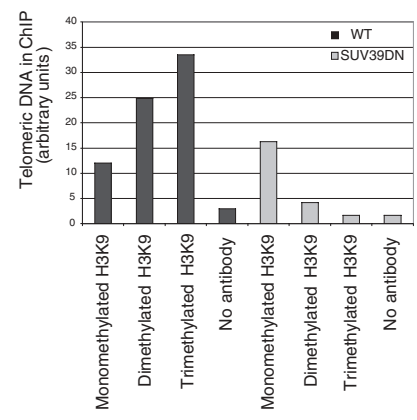

e

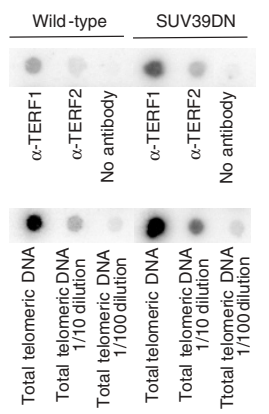

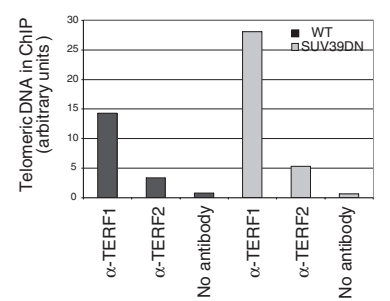

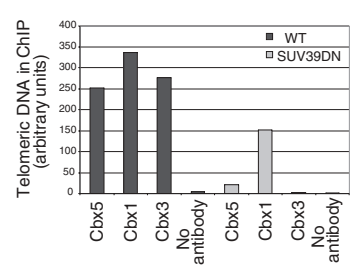

Figure 3 Loss of heterochromatin features at SUV39DN telomeres. (a) ChIP analyses were carried out in MEFs with the indicated antibodies. Quantification of the telomeric signal is shown also in the right panel. The same dot blot was probed with a minor satellite probe as control for binding to centromeric repeats. (b) ChIP analyses were carried out in ES cells with the indicated antibodies. Quantification of the telomeric signal is shown also in the right panel. (c) ChIP analyses were carried out in ES cells with antibodies against Cbx1, Cbx3 and Cbx5. Quantification of the telomeric signal is shown also in the right panel. (d) ChIP analyses were carried out in ES cells with the indicated antibodies against mono-, di- and trimethylated H3-Lys9. Quantification of the telomeric signal is shown also in the right panel. (e) ChIP analyses were carried out in MEFs with the indicated antibodies. 
Figure 4 Model for epigenetic regulation of telomere length. Mammalian telomeres contain features of constitutive heterochromatin, such as enrichment for diand trimethylated H3-Lys9 and binding of $\mathrm{Cbx} 1, \mathrm{Cbx} 3$ and $\mathrm{Cbx} 5$ (HP1). In the absence of the HMTases Suv39h1 and Suv39h2, diand trimethylation of H3-Lys9 is reduced and monomethylation of H3-Lys9 is increased at telomeres. Telomeric chromatin in SUV39DN cells also has less binding of $\mathrm{Cb} \times 3, \mathrm{Cb} \times 5$ and, to a lesser extent, $\mathrm{Cb} \times 1$. These changes in the composition of the telomeric chromatin in SUV39DN cells suggest a loss of heterochromatic features, which could explain the telomere elongation phenotype of these cells. The binding of a specific telomere-binding protein, TERF2, which is essential for telomere protection, was unchanged in SUV39DN cells, in agreement with a normal telomere capping function in these cells. TERF1, another telomere-specific binding protein, showed a slight increase in binding at SUV39DN telomeres, which may reflect the fact that these telomeres have an altered chromatin structure.

in telomere protection and telomere length control, respectively ${ }^{2,17}$. TERF2 was absent from centromeric repeats, indicating that the ChIP assay is specific (Fig. 3a). The abundance of TERF2 at telomeric repeats was similar in wild-type and SUV39DN cells (Fig. 3a,e), indicating that binding of TERF2 to telomeres is independent of the degree of H3-Lys9 methylation. Normal binding of TERF2 to SUV39DN telomeres is consistent with the normal capping function of the telomeres that we observed. TERF1 was slightly more abundant at SUV39DN telomeres than at control telomeres, which may reflect changes in the telomere structure ${ }^{18}$. Taken together, these results show that telomeric chromatin in SUV39DN cells is significantly altered in the absence of Suv39h1 and Suv39h2, resulting in loss of heterochromatic features (Fig. 3). Binding of TERF1 and TERF2, however, is preserved in these cells.

Our results show that methylation of H3-Lys9 by Suv39h1 and Suv39h2 occurs at mammalian telomeres and that it is important for telomere length regulation. Abrogation of Suv39h1 and Suv39h2 results in less di- and trimethylation of H3-Lys9 at telomeres, more monomethylation of H3-Lys9 and less binding of all three chromobox proteins (Fig. 4). These changes are substantial ( $~ 80 \%$ reduction in diand trimethylated H3-Lys9 and in binding of Cbx3 and Cbx5), suggesting that they affect most telomeres in the cell. Notably, these changes at the telomeric chromatin suggest loss of heterochromatic features at SUV39DN telomeres, which could explain the abnormal telomere elongation in these cells (Fig. 4). These findings imply that telomere length can be regulated by epigenetic factors, such as H3Lys9 methylation, linking for the first time histone modifications with telomere length regulation in mammalian cells. Suv39h1 and Suv39h2 are not the only HMTases responsible for methylation of H3-Lys9 in mammalian heterochromatic regions; therefore, other HMTases of the same family (SET domain-containing enzymes) ${ }^{19}$ may also contribute to the epigenetic regulation of telomere length in mammalian cells.

Finally, our findings imply that epigenetic errors could alter telomere length in mammals and help explain the differential resetting of telomere length in different cloned animals ${ }^{20,21}$. In addition,

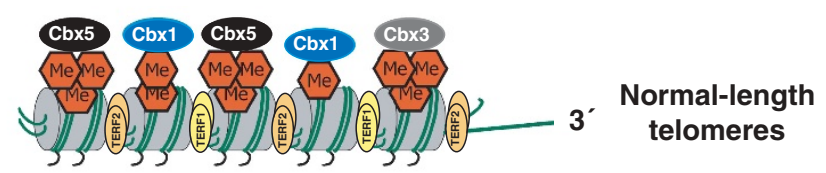

H3K9 (mono-, di- and trimethylated)
Cbx5, Cbx1, Cbx3
TERF1
TERF2

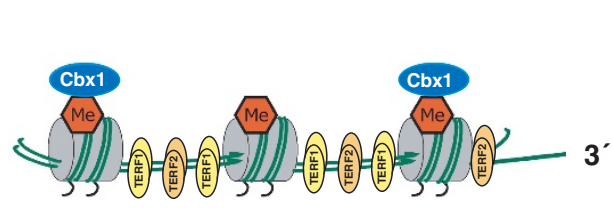

$\downarrow$ H3K9 (di-, trimethylated)

4 H3K9 (monomethylated)

$\downarrow \mathrm{Cbx} 5, \mathrm{Cbx} 3$

$\downarrow 50 \%$ decrease in $\mathrm{Cbx} 1$

Normal TERF2 binding

Slight increase in TERF1 binding

they provide a connection between the transcriptional silencing of genes near the telomeres and the regulation of telomere length in mammalian cells ${ }^{22,23}$, in agreement with findings in yeast that show a role for histone $\mathrm{H} 3$ methyltransferases in telomere silencing ${ }^{24}$. The epigenetic regulation of telomere length may also have an impact in pathologies of cancer and aging.

\section{METHODS}

Cells. Wild-type and SUV39DN MEFs and ES cells were on a mixed genetic background of 129/SV and C57BL/6J origin ${ }^{7,25}$. We prepared MEFs from wildtype and SUV39DN embryos at embryonic day 12.5 as described ${ }^{26}$. The first passage after MEF isolation was considered passage 1 .

Telomere length measurements: Q-FISH. We prepared metaphases for QFISH and hybridized them as described ${ }^{12}$. To correct for lamp intensity and alignment, we analyzed images from fluorescent beads (Molecular Probes) using the TFL-Telo program (gift from P. Lansdorp, Terry Fox Laboratory, Vancouver). Telomere fluorescence values were extrapolated from the telomere fluorescence of LY-R (R cells) and LY-S ( $\mathrm{S}$ cells) lymphoma cell lines of known lengths of 80 and $10 \mathrm{~kb}$ (ref. 27). There was a linear correlation $\left(\mathrm{r}^{2}=0.999\right)$ between the fluorescence intensity of the $\mathrm{R}$ and $\mathrm{S}$ telomeres with a slope of 38.6. We calculated the calibration-corrected telomere fluorescence intensity as described ${ }^{28}$. Images were captured using Leica Q-FISH software at $400 \mathrm{~ms}$ integration-time in a linear acquisition mode to prevent oversaturation of fluorescence intensity and recorded using a COHU CCD camera on a Leica Leitz DMRB fluorescence microscope. We used TFL-Telo software to quantify the fluorescence intensity of telomeres from ten metaphases of each data point. We captured the images of metaphases from different cell cultures on the same day, in parallel and blindly.

TRF analysis. We prepared cells in agarose plugs and carried out TRF analysis as described ${ }^{10}$.

Scoring of chromosomal abnormalities by Q-FISH. We scored the indicated numbers of metaphases from each ES cell or MEF culture for chromosomal aberrations by superimposing the telomere image on the DAPI chromosome image in the TFL-Telo software. End-to-end fusions can be two chromosomes fused by either their $\mathrm{p}$ arms (RT-like fusions) or their $\mathrm{q}$ arms (dicentrics). 
SKY. SKY analysis allowed identification of the chromosomes harboring very long telomeres as previously determined by Q-FISH. We generated painting probes for each chromosome from flow-sorted mouse chromosomes using sequence-independent DNA amplification. Labeling was done by incorporating four different dyes in a combination sequence that allows unique and differential identification of each chromosome. We prepared slides from fixative-stored material and hybridized and washed them using the SKY method according to the manufacturer's protocol (Applied Spectral Imaging). We counterstained chromosomes with DAPI. We captured and processed images as described ${ }^{29}$. We captured the indicated number of metaphases of each culture, analyzed them by SKY and scored chromosomal abnormalities as above.

Telomerase assay. We measured telomerase activity with a modified telomeric repeat amplification protocol as described ${ }^{10}$.

ChIP assay and telomere dot-blots. For ChIP analysis, we used $2 \times 10^{6} \mathrm{MEFs}$ or ES cells per condition. We added formaldehyde directly to tissue culture medium to a final concentration of $1 \%$ and incubated cultures for $10 \mathrm{~min}$ at room temperature on a shaking platform. We stopped the crosslinking by adding glycine to a final concentration of $0.125 \mathrm{M}$. We washed crosslinked cells twice with cold phosphate-buffered saline, scraped them and lysed them at a density of $5 \times 10^{6}$ cells $\mathrm{ml}^{-1}$ for $10 \mathrm{~min}$ at $4^{\circ} \mathrm{C}$ in $1 \%$ SDS, $50 \mathrm{mM}$ Tris- $\mathrm{HCl}(\mathrm{pH} 8.0)$ and $10 \mathrm{mM}$ EDTA containing protease inhibitors. We sonicated lysates to obtain chromatin fragments $<1 \mathrm{~kb}$ and centrifuged them for $15 \mathrm{~min}$ in a microfuge at room temperature. We diluted $400 \mu \mathrm{l}$ of lysate $1: 10$ with $1.1 \%$ Triton-X100, 2 mM EDTA, 150 $\mathrm{mM} \mathrm{NaCl}$ and $20 \mathrm{mM}$ Tris- $\mathrm{HCl}$ ( $\mathrm{pH}$ 8.0) containing protease inhibitors, precleared with 50\% salmon sperm DNA and protein A agarose slurry (Upstate). We incubated the fragments with $5 \mu \mathrm{l}$ of rabbit polyclonal antibody to TERF2 (SWE38; gift from S. West and M. Tarsounas, Cancer Research, UK); $8 \mu \mathrm{l} \mathrm{of} \mathrm{rab-}$ bit polyclonal antibody to TERF1 raised in our laboratory against full-length mouse TERF1 protein; $4 \mu \mathrm{g}$ of rabbit polyclonal antibody to branched methylated H3-Lys9 (refs. 7,30); rabbit polyclonal antibody to trimethylated H3-Lys9 (ref. 29); rabbit polyclonal antibody to trimethylated H3-Lys27 (ref. 29); preimmune serum; or no antibody at $4{ }^{\circ} \mathrm{C}$ overnight on a rotating platform. We carried out ChIP analysis with antibodies against mono-, di- and trimethylated H3-Lys9 and against $\mathrm{Cbx} 1, \mathrm{Cbx} 3$ and $\mathrm{Cbx} 5$ as described ${ }^{16}$. We then added salmon sperm DNA and protein A agarose beads $(60 \mu \mathrm{l})$ and incubated for $1 \mathrm{~h}$. We washed the immunoprecipitated pellets with $0.1 \%$ SDS, $1 \%$ Triton-X100, 2 mM EDTA, 20 $\mathrm{mM}$ Tris- $\mathrm{HCl}$ (pH 8.0) and $150 \mathrm{mM} \mathrm{NaCl}$ (one wash); 0.1\% SDS, $1 \%$ TritonX100, 2 mM EDTA, $20 \mathrm{mM}$ Tris-HCl (pH 8.0) and $500 \mathrm{mM} \mathrm{NaCl}$ (one wash); $0.25 \mathrm{M} \mathrm{LiCl}, 1 \%$ Nonidet P-40, $1 \%$ sodium deoxycholate, $1 \mathrm{mM}$ EDTA and 10 $\mathrm{mM}$ Tris-HCl, $\mathrm{pH} 8.0$ (one wash); and $10 \mathrm{mM}$ Tris- $\mathrm{HCl}(\mathrm{pH} 8.0)$ and $1 \mathrm{mM}$ EDTA (two washes). We then eluted the chromatin from the beads twice by incubation with $250 \mu \mathrm{l} \% \mathrm{SDS}$ and $0.1 \mathrm{M} \mathrm{NaHCO}_{3}$ during $15 \mathrm{~min}$ at room temperature with rotation. After adding $20 \mu \mathrm{l}$ of $5 \mathrm{M} \mathrm{NaCl}$, we reversed the crosslinks for $4 \mathrm{~h}$ at $65^{\circ} \mathrm{C}$. Samples were supplemented with $20 \mu \mathrm{l}$ of $1 \mathrm{M}$ Tris- $\mathrm{HCl}(\mathrm{pH} 6.5), 10$ $\mu \mathrm{l}$ of $0.5 \mathrm{M}$ EDTA, $20 \mu \mathrm{g}$ of RNase A and $40 \mu \mathrm{g}$ of proteinase $\mathrm{K}$ and incubated for $1 \mathrm{~h}$ at $45^{\circ} \mathrm{C}$. We recovered DNA by phenol-chloroform extraction and ethanol precipitation, slot-blotted it onto a Hybond $\mathrm{N}^{+}$membrane and hybridized it with a plasmid containing $1.6 \mathrm{~kb}$ of TTAGGG repeats (gift from $\mathrm{T}$. de Lange, Rockefeller University, USA). We quantified the signal with the ImageQuant software. For total telomeric DNA samples (Fig. 3e), we processed $20 \mu$ l of lysate with the rest of the samples at the step of reversing the crosslinks. We calculated the amount of telomeric DNA immunoprecipitated in each ChIP based on the signal relative to the corresponding total telomeric DNA signal. To determine histone methylation at centromeric major satellites, we used real-time PCR analysis (Supplementary Fig. $3 \mathbf{b}$ online) with specific primers (sequences available on request) under the following conditions: $94{ }^{\circ} \mathrm{C}$ for $3 \mathrm{~min}, 94^{\circ} \mathrm{C}$ for $30 \mathrm{~s}, 52{ }^{\circ} \mathrm{C}$ annealing for $30 \mathrm{~s}$. We ran the program on a Corbett Lightcycler using the Rotorgene software. Each reaction was run for 25 cycles until saturation was reached. The data in Supplementary Figure 3a,b online are represented as percentages of the input in the ChIP assays, which were done at least three times.

Note: Supplementary information is available on the Nature Genetics website.

\section{ACKNOWLEDGMENTS}

We thank S. Gonzalo and M. Serrano for critical reading of the manuscript and discussions and A.A.H.A. Derijck for generating SUV39DN ES cells. M.G.-C. is a predoctoral fellow from the Spanish Ministry of Science and Technology. A.H.F.M.P. is the recipient of a Marie Curie fellowship. Research in the laboratory of T.J. is supported by the Institute of Molecular Pathology through Boehringer Ingelheim and by grants from the Vienna Economy Promotion Fund, the European Union and the GEN-AU initiative, which is financed by the Austrian Ministry of Education, Science and Culture. Research at the laboratory of M.A.B. is funded by grants from the Spanish Ministry of Science and Technology, the Regional Government of Madrid and the European Union and by the Department of Immunology and Oncology.

\section{COMPETING INTERESTS STATEMENT}

The authors declare that they have no competing financial interests.

Received 30 September; accepted 17 November 2003

Published online at http://www.nature.com/naturegenetics/

1. Blackburn, E.H. Switching and signaling at the telomere. Cell 106, 661-673 (2001).

2. Chan, S.W.-L. \& Blackburn, E.H. New ways not to make ends meet: telomerase, DNA damage proteins and heterochromatin. Oncogene 21, 553-563 (2002).

3. De Lange, T. Protection of mammalian telomeres. Oncogene 21, 532-540 (2002).

4. Cenci, G., Siriaco, G., Raffa, G.D., Kellum, R. \& Gatti, M. The Drosophila HOAP protein is required for telomere capping. Nat. Cell. Biol. 1, 82-84 (2003).

5. Fanti, L., Giovinazzo, G., Berloco, M. \& Pimpinelli, S. The heterochromatin protein 1 prevents telomere fusions in Drosophila. Mol. Cell 2, 527-538 (1998).

6. Dubrana, K., Perrod, S. \& Gasser, S.M. Turning telomeres off and on. Curr. Opin. Cell Biol. 13, 281-289 (2001)

7. Peters, A.H. et al. Loss of the Suv39h histone methyltransferases impairs mammalian heterochromatin and genome stability. Cell 107, 323-337 (2001).

8. Jenuwein, T. \& Allis, C.D. Translating the histone code. Science 293, 1074-1080 (2001).

9. Lachner, M., O'Carrol, D., Rea, S., Mechtler, K. \& Jenuwein, T. Methylation of histone H3 lysine 9 creates a binding site for HP1 proteins. Nature 410, 116-120 (2001).

10. Blasco, M.A. et al. Telomere shortening and tumor formation by mouse cells lacking telomerase RNA. Cel/ 91, 25-34 (1997).

11. Hande, P.M., Samper, E., Lansdorp, P. \& Blasco, M.A. Telomere length dynamics in cultured cells from normal and telomerase null mice. J. Cell Biol. 144, 589-601 (1999).

12. Samper, E., Goytisolo, F., Slijepcevic, P., van Buul, P. \& Blasco, M.A. Mammalian Ku86 prevents telomeric fusions independently of the length of TTAGGG repeats and the G-strand overhang. EMBO Rep. 1, 244-252 (2000).

13. van Steensel, B., Smogorzewska, A. \& de Lange, T. TRF2 protects human telomeres from end-to-end fusions. Cel/ 92, 401-413 (1998)

14. Samper, E., Flores, J.M. \& Blasco, M.A. Restoration of telomerase activity rescues chromosomal instability and premature aging in $\mathrm{Terc}^{-/}$mice with short telomeres. EMBO Rep. 2, 800-807 (2001).

15. Hemann, M.T., Strong, M.A., Hao, L.Y. \& Greider, C.W. The shortest telomere, not average telomere length, is critical for cell viability and chromosome stability. Cell 107, 67-77 (2001)

16. Peters, A.H. et al. Partitioning and plasticity of repressive histone methylation states in mammalian chromatin. Mol. Cell (in the press).

17. Loayza, D. \& De Lange, T. POT1 as a terminal transducer of TRF1 telomere length control. Nature 424, 1013-1018 (2003).

18. Espejel, S. et al. Mammalian Ku86 mediates chromosomal fusions and apoptosis caused by critically short telomeres. EMBO J. 21, 2207-2219 (2002).

19. Jenuwein, T. Re-SET-ting heterochromatin by histone methyltransferases. Trends Cell Biol. 11, 266-273 (2001)

20. Shiels, P.G. et al. Analysis of telomere lengths in cloned sheep. Nature $\mathbf{3 9 9}$, 316-317 (1999)

21. Lanza, R.P. et al. Extension of cell life-span and telomere length in animals cloned from senescent somatic cells. Science 288, 665-669 (2000).

22. Baur, J.A., Zou, Y., Shay, J.W. \& Wright, W.E. Telomere position effect in human cells. Science 292, 2075-2077 (2001).

23. Koering, C.E. et al. Human telomeric position effect is determined by chromosomal context and telomeric chromatin integrity. EMBO Rep. 3, 1055-1061 (2002).

24. Ng, H.H. et al. Lysine methylation within the globular domain of histone H3 by Dot 1 is important for telomeric silencing and Sir protein association. Genes Dev. 16 1518-1527 (2002).

25. Lehnertz, B. et al. Suv39h-mediated histone H3 lysine 9 methylation directs DNA methylation to major satellite repeats at pericentric heterochromatin. Curr. Biol. 13, $1192-1200$ (2003).

26. O'Carroll, D. et al. Isolation and characterization of Suv39h2, a second histone H3 methyltransferase gene that displays testis-specific expression. Mol. Cell Biol. 20, 9423-9433 (2000)

27. Mcllrath, J. et al. Telomere length abnormalities in mammalian radiosensitive cells. Cancer Res. 61, 912-915 (2001).

28. Herrera, E. et al. Disease states associated with telomerase deficiency appear earlier in mice with short telomeres. EMBO J. 18, 2950-2960 (1999).

29. Espejel, S. et al. Mammalian Ku86 mediates chromosomal fusions and apoptosis caused by critically short telomeres. EMBO J. 21, 2207-2219 (2002).

30. Perez-Burgos, L. et al. Generation and characterization of methyl-lysine histone antibodies. Methods Enzymol. (in the press). 